\title{
Dynamical circle covering with homogeneous Poisson updating
}

\author{
Johan Jonasson ${ }^{*+1}$
}

April 3, 2008

\begin{abstract}
We consider a dynamical variant of Dvoretzky's classical covering problem of the unit circumference circle, where the centers of the arcs are updated according to independent Poisson processes of unit intensity. This dynamical model was introduced (in greater generality) in Jonasson and Steif (2008), where is was shown that when the length of the $n^{\prime}$ th $\operatorname{arc}$ is $\ell_{n}$ and we write $u_{n}:=\prod_{k=1}^{n}\left(1-\ell_{k}\right)$, then $\lim \inf _{n} n(\log n) u_{n}<\infty$ implies that the whole circle is a.s. covered at all times, whereas if $\sum_{n} e^{\ell_{1}+\ldots+\ell_{n}} /\left(n^{2} \log n\right)<\infty$, then a.s. there are times at which the circle is not fully covered.

In this paper we modify the former condition to $\lim \sup _{n} n u_{n}<\infty$. In particular this takes care of the natural border case $\ell_{n}=1 / n$; no exceptional times exist. More generally, with the parametrization $\ell_{n}=c / n$, there is no $c$ for which there are exceptional times for which the model behaves differently than for the static case.
\end{abstract}

AMS Subject classification : $60 \mathrm{~K} 99$

Key words and phrases: Circle coverings, exceptional times

Short title: Dynamical circle covering

\section{Introduction}

We first briefly introduce the classical static circle covering model. Let $C$ denote the circle with circumference 1 and consider a decreasing sequence $\left\{\ell_{n}\right\}_{n \geq 1}$ of positive numbers

\footnotetext{
${ }^{*}$ Chalmers University of Technology

${ }^{\dagger}$ Göteborg University

$\ddagger_{\text {http://www.math.chalmers.se/ jonasson }}$
} 
approaching 0 . Let $I_{n}$ be the open arc of $C$ of length $\ell_{n}$ with a center chosen uniformly at random independently of the other arcs. Let $E:=\limsup _{n} I_{n}$ and $F:=E^{c}$. By the the Borel-Cantelli Lemma, for each $x \in C, P(x \in E)=1$ if and only if $\sum_{n=1}^{\infty} \ell_{n}=$ $\infty$. Fubini's Theorem yields immediately that in this case $F$ has Lebesgue measure 0 a.s. Dvoretzky (1956) raised the question of whether in the $\sum_{n} \ell_{n}=\infty$ case it was possible that $F$ was nonempty and gave examples where this was indeed the case. There were a number of contributions to this question with the final result proved by Shepp (1972). (Kolmogorov's 0-1 law tells us that $P(F=\emptyset) \in\{0,1\}$.)

Theorem 1.1. (L. Shepp). $P(F=\emptyset)=1$ if and only if

$$
\sum_{n=1}^{\infty} \frac{e^{\ell_{1}+\ldots+\ell_{n}}}{n^{2}}=\infty .
$$

In particular if $\ell_{n}=c / n$ for all $n$, then $P(F=\emptyset)=1$ if and only if $c \geq 1$.

The special cases $\ell_{n}=c / n$ for a constant $c$ were known earlier, see Kahane (1959), Billard (1965) and Mandelbrot (1972). For a fuller introduction, see [5].

Jonasson and Steif (2008) extended the classical model by including time dynamics. Two different models were considered. In the first of these, the centers of the arcs perform independent Brownian motions on $C$, each with variance 1 . In the second model, one associates independent Poisson processes, or jump pocesses, with the different arcs, where the Poisson process associated with the $n$th arc has intensity $\ell_{n}^{-\alpha}$ for some parameter $\alpha \geq$ 0 . At the jump-times of the $n$th arc, $I_{n}$ is given a new center, chosen uniformly on $C$, independent of everything else. It was then asked, for each of these two models, if there are exceptional times at which we see different covering behavior from that which is seen in the earlier static model. Various results were shown. In the present paper, however, we will only focus on the latter model, i.e. the one with Poisson updating. Indeed, we will only be concerned with the perhaps most natural variant, namely with $\alpha=0$, i.e. each arc has updating intensity 1. In Jonasson and Steif (2008), the following result was shown. Here, of course, $E_{t}$ is the set of points of $C$ that are covered by infinitely many arcs at time $t$, and $F_{t}=E_{t}^{c}$.

Theorem 1.2. Consider the Poisson updating model with $\alpha=0$. Assume that there are constants $0<M_{0}<M_{1}<\infty$ such that for all $n, M_{0} / n<\ell_{n}<M_{1} / n$. Let $u_{n}:=$ $\prod_{k=1}^{n}\left(1-\ell_{k}\right)$. Then

(i) If $\liminf \operatorname{in}_{n} n(\log n) u_{n}<\infty$, then $P\left(\exists t \in[0,1]: F_{t} \neq \emptyset\right)=0$. 
(ii) If

$$
\sum_{n=1}^{\infty} \frac{e^{\ell_{1}+\ell_{2}+\ldots+\ell_{n}}}{n^{2} \log n}<\infty,
$$

then $P\left(\exists t \in[0,1]: F_{t} \neq \emptyset\right)=1$.

This result is of course satisfying in the sense that it proves that there are $\ell_{n}$ 's for which circle is fully covered at any fixed time but where there are exceptional times at which this fails. This e.g. happens for $\ell_{n}=1 / n-1 /(n \log n)$. On the other hand, it is unsatisfying since it does not, for the original and most natural parametrization $\ell_{n}=c / n$, tell us what happens for the critical case $c=1$. The point of this paper is to modify part (i) to the following result, which e.g. tells us that for $\ell_{n}=1 / n$, there are no exceptional times.

Theorem 1.3. If $\lim \sup _{n} n u_{n}<\infty$, then $P\left(\exists t \in[0,1]: F_{t} \neq \emptyset\right)=0$.

There is still a very narrow gap between what is covered by Theorem 1.2(ii) and Theorem 1.3, e.g. when $\ell_{n}=1 / n-1 /(n \log n \log \log n)$. It would of course be interesting to settle what happens for any $\left\{\ell_{n}\right\}$.

Remark on measurability. It is not immediately obvious that the set $\left\{\exists t: F_{t} \neq \emptyset\right\}$ is measurable. If the $\ell_{n}$ 's are such that $P(F \neq \emptyset)=1$ for the static case, then the complement of the interesting set is contained in a null set and hence measurable (after a completion of the probability space if necessary). We may thus assume that $P(F \neq \emptyset)=0$ for the static case. Now note first that

$$
\left\{\exists t: F_{t} \neq \emptyset\right\}=\left\{\exists t: \bigcup_{n} \bigcap_{m \geq n} I_{n}(t)^{c} \neq \emptyset\right\}=\bigcup_{n}\left\{\exists t: \bigcap_{m \geq n} I_{n}(t)^{c} \neq \emptyset\right\} .
$$

Hence it suffices to show that the set $\left\{\exists t: \cap_{n} I_{n}(t)^{c} \neq \emptyset\right\}$ is measurable. Letting $S_{n}:=$ $\left\{(x, t) \in C \times[0,1]: x \in I_{n}(t)^{c}\right\}$ and $S=\cap_{n} S_{n}$, this set can be written as $\{S \neq \emptyset\}$. Observe that by compactness,

$$
\left\{\cap_{n} \overline{S_{n}} \neq \emptyset\right\}=\bigcap_{N}\left\{\cap_{n=1}^{N} \overline{S_{n}} \neq \emptyset\right\}
$$

Now, the whole dynamical model can be defined in a straightforward way (as in Jonasson and Steif (2008), page 2-3) in terms of a set $\left\{X_{i j}\right\}_{i, j=1}^{\infty}$ of independent $C$-valued random variables (the center-points of the arcs) and a set $\left\{Y_{i j}\right\}_{i, j=1}^{\infty}$ of independent exponential random variables (the times between updatings). The set $\left\{\cap_{n=1}^{N} \overline{S_{n}} \neq \emptyset\right\}$ can then clearly be expressed as a countable union of events expressed in terms of these random variables. Therefore this set is measurable and hence so is $\left\{\cap_{n} \overline{S_{n}} \neq \emptyset\right\}$. Finally the set $S$ and $\cap_{n} \overline{S_{n}}$ may differ only on sets of the type $I_{n}^{c} \times\left\{t_{0}\right\}$ where $t_{0}$ is a jump time for some 
arc. The number of such sets is countable, and each of them is almost surely empty since $P(F \neq \emptyset)=0$ for the static case. Hence, possibly after a completion of the probability space, $\{S \neq \emptyset\}$ is measurable.

\section{Proof of Theorem 1.3}

Consider the static model. Let $\mu$ be Lebesgue measure on $C=\mathbb{R} / \mathbb{Z}$, let $U=\cap_{n=1}^{\infty} I_{n}^{c}$, i.e. the set of points of $C$ that are not covered by any arc, and let $U_{n}=\cap_{k=1}^{n} I_{k}^{c}$. It is easily seen that $P(F \neq \emptyset)=1$ if and only if $P(U \neq \emptyset)=\lim _{n \rightarrow \infty} P\left(U_{n} \neq \emptyset\right)=$ $\lim _{n \rightarrow \infty} P\left(\mu\left(U_{n}\right)>0\right)>0$. The key ingredient in the proof of Theorem 1.1 is the fact that $\mathbb{E}\left[\mu\left(U_{n}\right) \mid U_{n} \neq \emptyset\right] \geq \frac{1}{2} \mathbb{E}\left[\mu\left(U_{n}\right) \mid 0 \in U_{n}\right]$. This follows from the lemma on page 146 of Kahane (1985) in the following way.

$$
\begin{aligned}
2 \mathbb{E}\left[\mu\left(U_{n}\right) \mid U_{n} \neq \emptyset\right]= & \frac{\mathbb{E} \mu\left(U_{n}\right)}{\frac{1}{2} P\left(\mu\left(U_{n}\right)>0\right)} \geq \frac{\mathbb{E} \mu\left(U_{n}\right)}{P\left(\mu\left(U_{n} \cap\left[0, \frac{1}{2}\right]\right)>0\right)} \\
& \geq \mathbb{E}\left[\mu\left(U_{n}\right) \mid 0 \in U_{n}\right]
\end{aligned}
$$

where the last inequality follows from the lemma applied with $\epsilon=1 / 2$. Hence, with $A_{x}:=\left\{x \in U_{n}\right\}$,

$$
\begin{gathered}
P\left(U_{n} \neq \emptyset\right)=\frac{\mathbb{E} \mu\left(U_{n}\right)}{\mathbb{E}\left[\mu\left(U_{n}\right) \mid U_{n} \neq \emptyset\right]} \leq \frac{2 \mathbb{E} \mu\left(U_{n}\right)}{\mathbb{E}\left[\mu\left(U_{n}\right) \mid 0 \in U_{n}\right]} \\
=\frac{2 P\left(A_{0}\right)}{\int_{C} P\left(A_{x} \mid A_{0}\right) d x}=\frac{2 P\left(A_{0}\right)^{2}}{\int_{C} P\left(A_{x} \cap A_{0}\right) d x},
\end{gathered}
$$

where the last two equalities follow from Fubini's Theorem.

Now consider the dynamical model on the time interval $[0, h]$. Let, for each $n, Z_{n}=$ $Z_{n}(h)$ be the indicator that arc $I_{n}$ does not update during the time interval $[0, h]$. Let $I_{n}^{\prime}=Z_{n} I_{n}(t)$ and let $U_{n}^{\prime}=\cap_{k=1}^{n}\left(I_{n}^{\prime}\right)^{c}$ and $U^{\prime}=\cap_{n} U_{n}^{\prime}$. Then clearly, if $U_{n}^{\prime}$ is empty, then so is $U_{n}(t)$ for every $t \in[0, h]$. Now, given the $Z_{n}$ 's, the $U_{n}^{\prime}$ 's correspond to a static model with some of the original arcs removed. Hence, putting $A_{x}^{\prime}:=\left\{x \in U_{n}^{\prime}\right\}$ and writing $P_{Z}$ for conditional probability given $Z_{1}, Z_{2}, \ldots$, we have

$$
P_{Z}\left(\mu\left(U_{n}^{\prime}\right)>0\right) \leq C_{2} \frac{P_{Z}\left(A_{0}^{\prime}\right)^{2}}{\int_{C} P_{Z}\left(A_{x}^{\prime} \cap A_{0}^{\prime}\right) d x} .
$$

We also have

$$
P_{Z}\left(A_{x}^{\prime} \cap A_{0}^{\prime}\right)=\prod_{k=1}^{n}\left(1-2 \ell_{k}+\left(\ell_{k}-x\right)^{+}\right)^{Z_{k}} .
$$


Observe that for any $a \in(0,1 / 2]$,

$$
\begin{gathered}
1-2 \ell_{k}+a>\left(1-\ell_{k}\right)^{2}+a>\left(1-\ell_{k}\right)^{2}(1+a) \\
>\left(1-\ell_{k}\right)^{2}\left(e^{a}-a^{2}\right)>\left(1-\ell_{k}\right)^{2} e^{a}\left(1-a^{2}\right) .
\end{gathered}
$$

Since $M_{0} / k<\ell_{k}<M_{1} / k$, it follows that

$$
P_{Z}\left(A_{x}^{\prime} \cap A_{0}^{\prime}\right)>\prod_{k=1}^{n}\left(\left(1-\ell_{k}\right)^{2} e^{\left(\ell_{k}-x\right)^{+}}\left(1-\ell_{k}^{2}\right)\right)^{Z_{k}}=\text { const } P_{Z}\left(A_{0}^{\prime}\right)^{2} e^{\sum_{k=1}^{n} Z_{k}\left(\ell_{k}-x\right)^{+}},
$$

where the constant is independent of $n$. By the condition $M_{0} / n<\ell_{n}<M_{1} / n$,

$$
\operatorname{Var} \sum_{n=1}^{\infty} Z_{n} \ell_{n}=\left(\sum_{n=1}^{\infty} \ell_{n}^{2}\right) \operatorname{Var} Z_{1}<\infty
$$

Therefore Chebyshev's inequality and the fact that $\mathbb{E} Z_{k}=e^{-h}>1-h$ imply that

$$
\sum_{k=1}^{n} Z_{k}\left(\ell_{k}-x\right)^{+}>\sum_{k=1}^{\left\lfloor M_{0} / x\right\rfloor \wedge n} Z_{k} \ell_{k}-M_{1}>(1-h) \sum_{k=1}^{\left\lfloor M_{0} / x\right\rfloor} \ell_{k}-M_{1}
$$

with probability tending to 1 as $n \rightarrow \infty$. Hence

$$
P\left(\mu\left(U_{n}^{\prime}\right)>0\right)<o(1)+\text { const }\left(\int_{C} e^{(1-h) \sum_{k=1}^{\left\lfloor M_{0} / x\right\rfloor} \ell_{k}-M_{1}} d x\right)^{-1} .
$$

Since $\lim \sup _{n} n u_{n}<\infty$, we have $\sup _{n} n u_{n}>M$ for some constant $M$. Therefore $e^{\ell_{1}+\ldots+\ell_{n}}>M^{-1} n$ and hence

$$
\int_{C} e^{(1-h) \sum_{k=1}^{\left\lfloor M_{0} / x\right\rfloor} \ell_{k}-M_{1}} d x>\text { const } \int_{0}^{1} x^{-(1-h)} d x=\text { const } \cdot h^{-1} .
$$

Thus

$$
P\left(\mu\left(U_{n}^{\prime}\right)>0\right)<o(1)+C h
$$

for a constant $C$ independent of $n$, and hence, by letting $n \rightarrow \infty$,

$$
P\left(\mu\left(U^{\prime}\right)>0\right)<C h .
$$

Thus, with $B(h)=\{\exists t \in[0, h]: U(t) \neq \emptyset\}$, we have $P(B(h))<C h$. This entails that, for any positive integer $k$, the expected number of time intervals $[(j-1) / k, j / k)$, $j=1,2, \ldots, k$ that contain a time point $t$ for which $U(t)$ occurs, is bounded by $C$. Putting $N_{n}$ for the number of such time intervals and $T$ for the set of $t$ 's for which $U(t)$ occurs, it is easy to see that $|T| \leq \liminf _{n} N_{n}$. Hence by Fatou's Lemma, $T$ is a.s. finite. However, since the process studied (i.e. the whole dynamical circle covering model) is a reversible Markov process, we conclude, by combining Theorem 6.7 in Getoor and Sharp (1984) and (2.9) in Fitzsimmons and Getoor (1988), that $T$ is a.s. empty.

Acknowledgments: Research partly supported by the Swedish Research Council. 


\section{References}

[1] Billard, P., Séries de Fourier aléatoirement bornées, continues, uniformément convergentes Ann. Sci. École Norm. Sup. (3) 82, 1965, 131-179.

[2] Dvoretzky, A., On covering a circle by randomly placed arcs Proc. Nat. Acad. Sci. U.S.A. 42, 1956, 199-203.

[3] Fitzsimmons P.J. and Getoor R.K., On the potential theory of symmetric Markov processes. Math. Ann. 281, 1988, 495-512.

[4] Getoor, R.K. and Sharp, M.J., Naturality, standardness, and weak duality for Markov processes. Zeits. Wahr. verw. Gebiete 67, 1984, 1-62.

[5] Jonasson, J. and Steif, J., Dynamical models for circle covering: Brownian motion and Poisson updating, Ann. Probab. 67, 2008, 739-764.

[6] Kahane, J.P., Sur le recouvrement d'un cercle par des arcs disposés au hasard $C$. R. Acad. Sci. Paris 248, 1959, 184-186.

[7] Kahane, J. P. (1985) Some random series of functions Second edition, Cambridge University Press: Cambridge.

[8] Mandelbrot, B., On Dvoretzky coverings for the circle Z. Wahrscheinlichkeitstheorie und Verw. Gebiete 22, 1972, 158-160.

[9] Shepp, L. A., Covering the circle with random arcs Israel J. Math. 11, 1972, 328345. 